\title{
Nanoscale
}

Check for updates

Cite this: Nanoscale, 2021, 13, 15763

\section{Interfacial magnetism in a fused superatomic cluster $\left[\mathrm{Co}_{6} \mathrm{Se}_{8}\left(\mathrm{PEt}_{3}\right)_{5}\right]_{2} \uparrow$}

\author{
Dinesh Bista, (D) Turbasu Sengupta, (D) Arthur C. Reber (D) and Shiv N. Khanna (D)*
}

An isolated $\mathrm{CO}_{6} \mathrm{Se}_{8}\left(\mathrm{PEt}_{3}\right)_{6}$ cluster is non-magnetic; however, we find that a magnetic unit can be formed by fusing two $\mathrm{Co}_{6} \mathrm{Se}_{8}\left(\mathrm{PEt}_{3}\right)_{5}$ superatoms into a $\left[\mathrm{CO}_{6} \mathrm{Se}_{8}\left(\mathrm{PEt}_{3}\right)_{5}\right]_{2}$ dimer. Theoretical studies indicate that the dumbbell-shaped $\left[\mathrm{CO}_{6} \mathrm{Se}_{8}\left(\mathrm{PEt}_{3}\right)_{5}\right]_{2}$ dimer has a spin moment of $2 \mu_{\mathrm{B}}$, and the spin density is primarily localized at the interfacial Co-sites where two clusters are fused into a dimer. The dimer has a low ionization energy of $4.17 \mathrm{eV}$, allowing the dimer to donate charge to $C_{70}$ during the formation of a cluster assembled material, as seen in recent experiments by Nuckolls and co-workers. The donation of charge causes the dimer's magnetic moment to drop from $2 \mu_{\mathrm{B}}$ to $1 \mu_{\mathrm{B}}$. We hypothesize that adding electrons to the dimer, such as doping impurities to the crystal lattice, may enhance the magnetic moment by neutralizing the charged cluster. This reveals a strategy for stabilizing magnetic moments in ligated cluster assemblies.

Received 8th February 2021, Accepted 10th September 2021 DOI: $10.1039 / \mathrm{d} 1 \mathrm{nr} 00876 \mathrm{e}$ rsc.li/nanoscale
The attached ligands generally protect the clusters and promote the formation of the ionic solids; however, the ligands may also lead to other outcomes. For example, $\mathrm{PEt}_{3}$ ligands quench the magnetic moment of the transition metal atoms, while the unligated metal cores usually possess a higher magnetic moment. ${ }^{24}$ Thus, it is important to ask if there is a way to protect the metal-chalcogenide cluster with ligands while also stabilizing the respective magnetic state. In this work, we show that by fusing two ligated superatomic clusters, one can form a dimer with a magnetic moment localized at the interface between the fused clusters. ${ }^{25-28}$ We demonstrate this intriguing possibility by considering the metal-chalcogenide cluster-core of $\mathrm{Co}_{6} \mathrm{Se}_{8}$ with $\mathrm{PEt}_{3}$ ligands. The addition of six $\mathrm{PEt}_{3}$ ligands to the Co-sites quenches the spin magnetic moment of the cluster.

In contrast, removing the $\mathrm{PEt}_{3}$ ligands can restore the spin moment. However, the unligated cluster becomes unstable. One way to overcome this problem is to join two $\mathrm{Co}_{6} \mathrm{Se}_{8}\left(\mathrm{PEt}_{3}\right)_{5}$ clusters into a composite $\left[\mathrm{Co}_{6} \mathrm{Se}_{8}\left(\mathrm{PEt}_{3}\right)_{5}\right]_{2}$ unit through covalent bonding between Co and Se sites on different clusters. Here we show that such a composite cluster has a finite spin moment localized at the interfacial sites, thus offering a unique opportunity for a tunable spin magnetic moment in a ligand protected cluster. Additionally, these magnetic units can also be arranged into a magnetic cluster assembly.

Our investigations are inspired by the recent experimental work by Nuckolls et al. ${ }^{29}$ who have stabilized metal-chalcogenide dimers, $\left[\mathrm{Co}_{6} \mathrm{Se}_{8}\left(\mathrm{PEt}_{3}\right)_{5}\right]_{2}$ with $\mathrm{C}_{70}{ }^{-}$dimers forming a cluster assembled solid. As pointed out by Nuckolls et $a .^{29}$ the dimers' elongated shapes allow the facile synthesis of novel solids that pack these dumbbell-shaped composite metal-chal- 
cogenide clusters with other dumbbell-shaped $\mathrm{C}_{140}$ counterions to form stable anisotropic solids. The toluene solvent fills the space between the dumbbell-shaped chalcogenide-dimers and $\mathrm{C}_{140}$. Furthermore, the shape allows the generation of multiple assemblies with differing atomic arrangements of these ionic cluster solids formed from $\left[\mathrm{Co}_{6} \mathrm{Se}_{8}\left(\mathrm{PEt}_{3}\right)_{5}\right]_{2}{ }^{+}$cations and $\mathrm{C}_{140}{ }^{2-}$ anions. In addition to the investigation of the magnetic character, we also analyze the role of charge transfer on the stability of the cluster assembled solid. We find large cohesive energy of the cluster assembled material demonstrating a significant stabilization during the formation of the material. This stabilization is caused by charge transfer from the metalchalcogenide dimers to the fullerenes. Hence, the material is stabilized via ionic bonding. The nature of electronic states characterizing the HOMO (highest occupied molecular orbital) and LUMO (lowest unoccupied molecular orbital) was analyzed to understand how this charge transfer affects the spin magnetic moment of the metal-chalcogenide dimers.

\section{Results}

\subsection{Effect of ligands on the magnetic properties of a $\mathrm{Co}_{6} \mathrm{Se}_{8}\left(\mathrm{PEt}_{3}\right)_{n}(\boldsymbol{n}=0-6)$ cluster and dimer formation}

We present a brief review of the ligand effects on $\mathrm{Co}_{6} \mathrm{Se}_{8}$ cluster's spin-magnetic moment. The ground-state structure of $\mathrm{Co}_{6} \mathrm{Se}_{8}$ consists of an octahedron of $6 \mathrm{Co}$-sites with faces decorated with 8 Se-atoms, as shown in Fig. 1a. The cluster has a spin magnetic moment of $10 \mu_{\mathrm{B}}$. The average Co-Se bond is

(a)
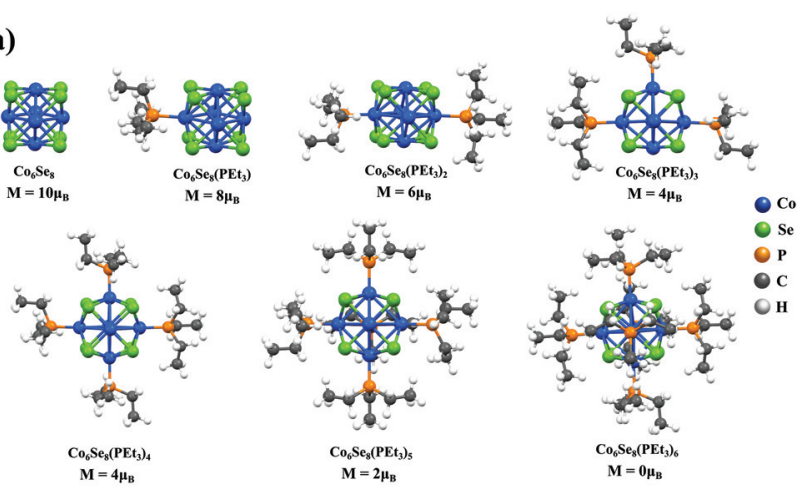

(b)

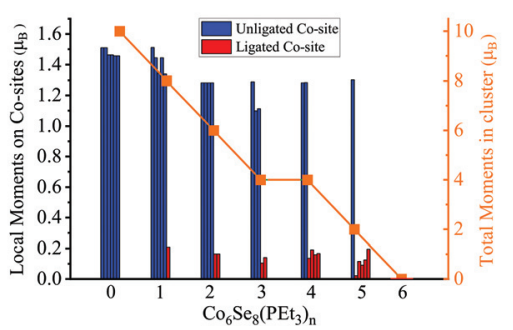

Fig. 1 (a) Ground state structures of $\mathrm{Co}_{6} \mathrm{Se}_{8}\left(\mathrm{PEt}_{3}\right)_{n}(n=0-6)$. The net magnetic moments $\left(\mu_{\mathrm{B}}\right)$ for each system are also provided. (b) A representative plot of the local magnetic moments of the unligated and ligated $\mathrm{Co}$ sites for $\mathrm{Co}_{6} \mathrm{Se}_{8}\left(\mathrm{PEt}_{3}\right)_{n}(n=0-6)$. The total magnetic moments of each clusters are also plotted in orange. around $2.39 \AA$, and each Co-site carries a local spin magnetic moment of approximately $1.5 \mu_{\mathrm{B}}$. All cluster structures were fully optimized without constraints. The successive additions of $\mathrm{PEt}_{3}$ ligands decrease the total magnetic moment monotonically, thereby completely quenching the magnetic moment for the fully ligated cluster, as seen in Fig. 1b. The local spinmoments reduce drastically after the ligation on the Co-sites, also seen in Fig. 1b. The magnetic moments on the Co-sites decrease from $1.5 \mu_{\mathrm{B}}$ to $0 \mu_{\mathrm{B}}$ once the $\mathrm{PEt}_{3}$ ligand is attached. $\mathrm{A}$ detailed electronic structure investigation reveals that the ligand states bind with the d-states of the Co-sites that lead to a filling of minority states resulting in a decrease in the local moment, as shown in our previous work. ${ }^{24}$

These results suggest that one way to create a magnetic cluster is to remove one or more ligands; however, losing the ligand exposes the metal-sites to reactants. Such unligated species are likely to be transient. One strategy for stabilizing such magnetic clusters is to fuse two ligated metal-chalcogenide clusters where the exposed metal ions bind to the chalcogen on the other cluster, making the system stable. When two $\mathrm{Co}_{6} \mathrm{Se}_{8}\left(\mathrm{PEt}_{3}\right)_{5}$ clusters, each with one missing ligand, combine through the unligated Co-sites of one cluster with the Se-sites of the other, they form a composite superatomic dimer $\left[\mathrm{Co}_{6} \mathrm{Se}_{8}\left(\mathrm{PEt}_{3}\right)_{5}\right]_{2}$, as shown in Fig. 2. This dimer could result in a magnetic species with no exposed metal sites and enhanced stability. The ligated dimer is protected from exposure; however, the lack of a closed electronic shell and numerous phosphine ligands means that such dimers will likely be excellent charge donors and become cations when forming the cluster assembled solids.

We investigated the atomic and electronic structure of $\left[\mathrm{Co}_{6} \mathrm{Se}_{8}\left(\mathrm{PEt}_{3}\right)_{5}\right]_{2}$ using theoretical methods. Fig. 2 shows the dumbbell-shaped ground state structure of $\left[\mathrm{Co}_{6} \mathrm{Se}_{8}\left(\mathrm{PEt}_{3}\right)_{5}\right]_{2}$,
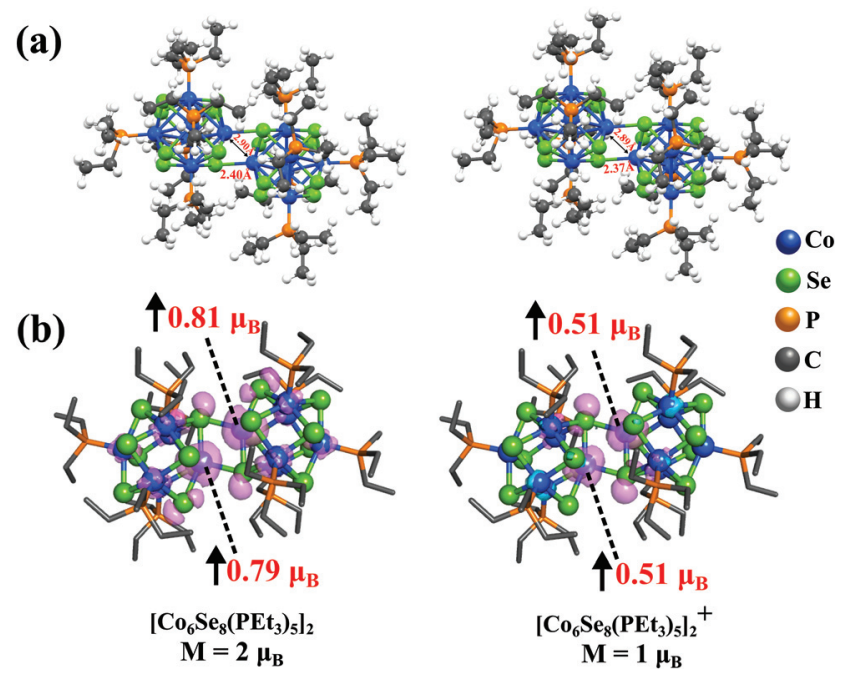

Fig. 2 (a) Optimized ground state structures and (b) spin density isosurface plot of the neutral and cationic $\left[\mathrm{Co}_{6} \mathrm{Se}_{8}\left(\mathrm{PEt}_{3}\right)_{5}\right]_{2}$ dimer. The value of spin magnetic moment at the interfacial Co-atoms are given in red font and the direction of the spin is shown by black arrow. 
which has a spin magnetic moment of $2 \mu_{\mathrm{B}}$. The Adiabatic Ionization Potential (AIP) of the dimer is only $4.17 \mathrm{eV}$, slightly less than that of a $\mathrm{K}$ atom, ${ }^{30}$ showing that it is a suitable donor. Since the cluster is expected to donate charge while forming an ionic solid, we also calculated the spin magnetic moment of the cationic species and found it to be $1 \mu_{\mathrm{B}}$. The spin densities for both neutral and cationic dimers are localized at the interfacial Co-atoms that lie at the junction between the fused clusters without any $\mathrm{PEt}_{3}$ ligand attached to them, as seen in the isosurfaces in Fig. 2b. The localization of the spin densities at the interface is due to the presence of dangling bonds on the Co atoms at the junction whose spin magnetic moment is quenched when ligated by the phosphine, but not by the Se of the adjacent cluster. For neutral dimer, Co-atoms at the junction have local spin moments of $0.81 \mu_{\mathrm{B}}$ and $0.79 \mu_{\mathrm{B}}$, while the other 10 Co-atoms contribute small spin moments to make a total $2 \mu_{\mathrm{B}}$ magnetic moment. Thus, we have a magnetic moment on the unligated Co-sites at the interface while still protecting the interfacial Co-atoms from the reactivity.

To gain more insight into the nature of states contributing to the moment, we show in Fig. 3, the one-electron levels in the neutral and cationic dimers. The ground state of the neutral cluster is marked by a pair of d-states $a_{1}$ and $a_{2}\left(D_{y z} /\right.$ $\mathrm{D}_{x z}$ and $\mathrm{D}_{z^{2}}$ ) in the majority spin states that are unoccupied in the minority spin states (b2 and b3), as shown in Fig. 3a. These states correspond to the d-states of the interfacial Coatoms and are responsible for the spin magnetic moment of $2 \mu_{\mathrm{B}}$. The HOMO level is in the majority spin, while the LUMO level is in the minority manifold. However, when the dimer becomes cationic, the HOMO level shifts to the minority spin channel, and the LUMO level shifts to the majority spin states, as shown in Fig. 3b. We also analyzed the electronic states to examine the stability of clusters. The neutral and cationic species are marked by HOMO-LUMO gaps of 0.35 and $0.30 \mathrm{eV}$, respectively.

Additionally, we found that the magnetic moment of the dimer with +2 oxidation state is also $2 \mu_{\mathrm{B}}$. It is interesting to note that both neutral and +2 state has a magnetic moment of $2 \mu_{\mathrm{B}}$, while the cationic dimer has the spin magnetic moment of $1 \mu_{\mathrm{B}}$. Since a pair of unfilled minority states characterize the neutral species, one may ask if filling those states would complete the electronic shell and lead to an electronically stable cluster. To explore this possibility, we investigated the electronic structure of a $\left[\mathrm{Co}_{6} \mathrm{Se}_{8}\left(\mathrm{PEt}_{3}\right)_{5}\right]_{2}{ }^{2-}$ cluster. The cluster is found to be non-magnetic with electronic shell-closure and a larger HOMO-LUMO gap of $0.53 \mathrm{eV}$, indicative of an electronically stable species, as shown in ESI Fig. $1 . \dagger$

\subsection{Cluster assembled solid}

As mentioned earlier, Nuckolls et $a .^{29}$ have recently synthesized a cluster assembled solid by combining $\left[\mathrm{Co}_{6} \mathrm{Se}_{8}\left(\mathrm{PEt}_{3}\right)_{5}\right]_{2}$ dimers with $\mathrm{C}_{140}$ motifs composed of twolinked $\mathrm{C}_{70}$ clusters. ${ }^{29}$ Both $\left[\mathrm{Co}_{6} \mathrm{Se}_{8}\left(\mathrm{PEt}_{3}\right)_{5}\right]_{2}$ and $\mathrm{C}_{140}$ are observed to have dumbbell-shaped structures, and unfilled interstitial space is filled by toluene solvent molecules. They

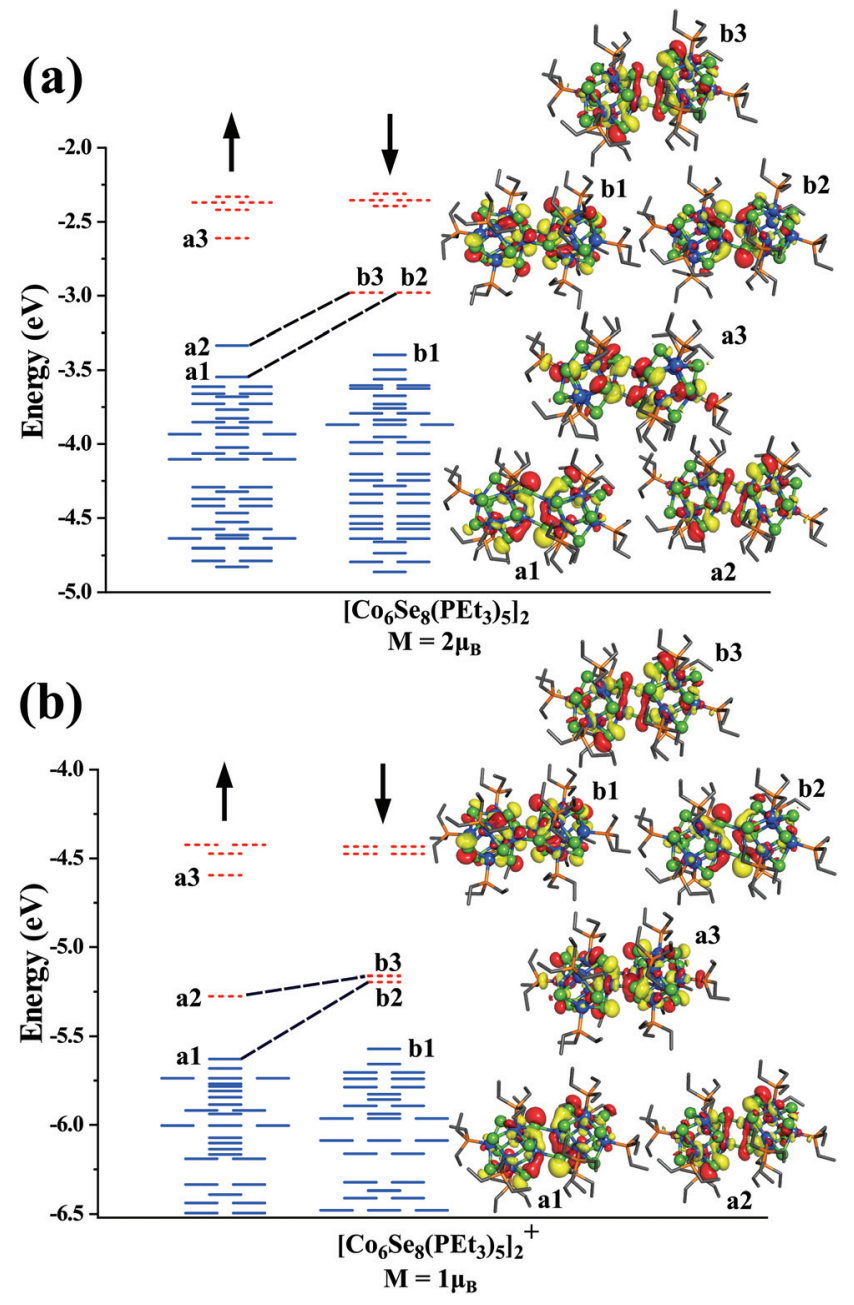

Fig. 3 The one-electronic energy levels of (a) neutral and (b) cationic $\left[\mathrm{CO}_{6} \mathrm{Se}_{8}\left(\mathrm{PEt}_{3}\right)_{5}\right]_{2}$ dimer. The blue (solid) and red (dotted) lines indicate occupied and unoccupied levels, respectively. The black (dashed) lines show the correspondence between the majority and the minority spin states. The isosurfaces of a few selected orbitals are also shown.

have synthesized three different cluster-assembled solids made from $\left[\mathrm{Co}_{6} \mathrm{Se}_{8}\left(\mathrm{PEt}_{3}\right)_{5}\right]_{2}$ and the $\mathrm{C}_{70}$ dimers $\left(\mathrm{C}_{140}\right)$, using different stoichiometry of toluene molecules. Initially, we start with investigating the redox properties of the cluster building blocks to understand the formation of this cluster assembled solid. The $\left[\mathrm{Co}_{6} \mathrm{Se}_{8}\left(\mathrm{PEt}_{3}\right)_{5}\right]_{2}$ dimers have a low AIP of $4.17 \mathrm{eV}$ and donate charge to the $\mathrm{C}_{70}$, which has a large electron affinity of $2.86 \mathrm{eV}$, as shown in ESI Fig. $2 . \dagger$ The $1 \mathrm{e}^{-}$energy levels of the $\left[\mathrm{Co}_{6} \mathrm{Se}_{8}\left(\mathrm{PEt}_{3}\right)_{5}\right]_{2}$ dimer and $\mathrm{C}_{70}$ are plotted side by side in ESI Fig. $3 \dagger$ indicate that the HOMO of the $\left[\mathrm{Co}_{6} \mathrm{Se}_{8}\left(\mathrm{PEt}_{3}\right)_{5}\right]_{2}$ dimer is higher than the LUMO of the $\mathrm{C}_{70}$.

Hence, the electron transfer from the dimer to the $\mathrm{C}_{70}$ is energetically favorable. The radical $\mathrm{C}_{70}{ }^{-}$anions formed have HOMO-LUMO gaps of only $0.12 \mathrm{eV}$ and are highly reactive. Hence, the $\mathrm{C}_{70}{ }^{-}$anions combine to form the dimer $\mathrm{C}_{140}{ }^{2-}$, that has a closed electronic shell and a large HOMO-LUMO gap of $0.73 \mathrm{eV}$, as shown in ESI Fig. $4 . \dagger$ 
We next examined the electronic structure of one of the crystal structures from the experiment ${ }^{29}$ to understand how the electronic structure of the metal chalcogenide-dimers evolves as they form the observed cluster-assembled solid. We studied the crystal structure of the solid with the stoichiometry of $\left.\left\{\left[\mathrm{Co}_{6} \mathrm{Se}_{8}\left(\mathrm{PEt}_{3}\right)_{5}\right]_{2} \text { (Toluene }\right)_{2} \mathrm{C}_{140}\right\}_{2}$ in the unit cell, and the details of the calculations are noted in the Methods section. The crystal structure was fully optimized without constraints. The shortest distance between atoms in the nearest $\mathrm{C}_{140}$ dimers is found to be $6.1 \AA$ A. Fig. 4 shows the unit cell and an isosurface of the electron spin density. Note that the spin density is localized on the interface between the fused clusters, as seen in Fig. $4 \mathrm{~b}$. The magnetic moment on each dimer is found to be $1 \mu_{\mathrm{B}}$; however, the coupling between the four dimers in the unit cell is antiferromagnetic. This antiferromagnetic arrangement is only $0.03 \mathrm{eV}$ more stable than the ferromagnetic state, which displays the magnetic moment of $1 \mu_{\mathrm{B}}$ per dimer or $4 \mu_{\mathrm{B}}$ per unit cell. The schematic of the antiferromagnetic and ferromagnetic couplings between the dimers in the solid is given in ESI Fig. 5. $\dagger$ Besides the energies being very close for the antiferromagnetic and ferromagnetic couplings, the bandgap energies are also almost equal with the values of $0.24 \mathrm{eV}$ for each. We know that the spin magnetic moments of the neutral and cationic dimers are $2 \mu_{\mathrm{B}}$ and $1 \mu_{\mathrm{B}}$, respectively, from our free cluster calculations. Hence, the

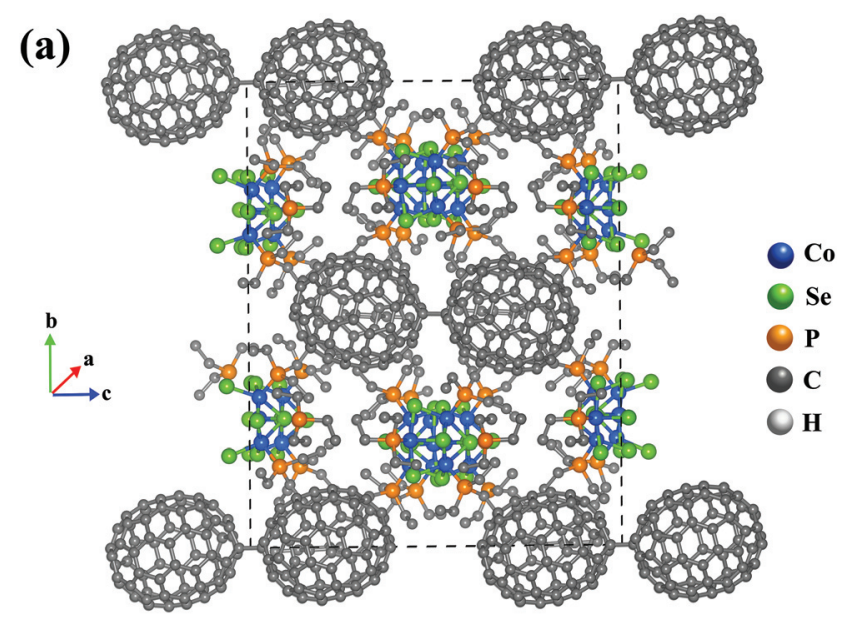

(b)

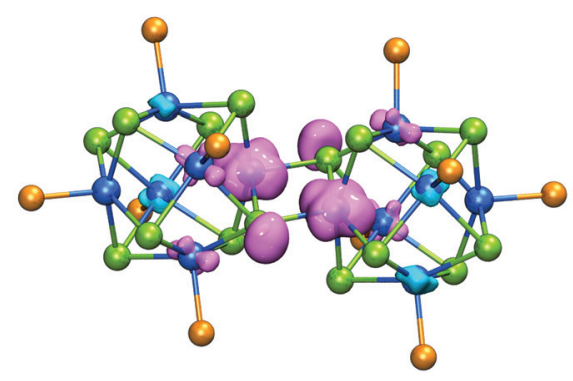

Fig. 4 (a) The optimized unit cell structure of $\left.\left\{\left[\mathrm{Co}_{6} \mathrm{Se}_{8}\left(\mathrm{PEt}_{3}\right)_{5}\right]_{2} \text { (Toluene }\right)_{2} \mathrm{C}_{140}\right\}_{2}$ crystal. (b) The spin density isosurface of the dimer unit. dimers in the solid with $1 \mu_{\mathrm{B}}$ can be best described as cations, $\left[\mathrm{Co}_{6} \mathrm{Se}_{8}\left(\mathrm{PEt}_{3}\right)_{5}\right]_{2}{ }^{+}$. To confirm this, we did a Bader analysis of the charge density and found a charge transfer of a $0.81 e^{-}$ from each $\left[\mathrm{Co}_{6} \mathrm{Se}_{8}\left(\mathrm{PEt}_{3}\right)_{5}\right]_{2}$-dimer units to the $\mathrm{C}_{140}$ units, as seen in Table 1. It confirms that the dimers are in the cationic state, and the $\mathrm{C}_{140}$ are also best described as $\mathrm{C}_{140}{ }^{2-}$. These theoretical calculations verify the experimental inference of charge transfer between the dimers and the fullerene units. ${ }^{29}$ The stabilization of the cluster assembly due to charge transfer is confirmed by an energy analysis that gives the cohesive energy of the unit cell to be $26.68 \mathrm{eV}$. The cohesive energy is calculated by eqn (1),

$$
E_{\text {cohesive }}=E_{\text {solid }}-4 E_{\left[\mathrm{Co}_{6} \mathrm{Se}_{8}\left(\mathrm{PEt}_{3}\right)_{5}\right]_{2}}-4 E_{\mathrm{C}_{70}}-4 E_{\mathrm{Tol}}
$$

$E_{\text {solid }}, E_{\left[\mathrm{Co}_{6} \mathrm{Se}_{8}\left(\mathrm{PEt}_{3}\right)_{5} l_{2}\right.}, E_{\mathrm{C}_{70}}$, and $E_{\mathrm{Tol}}$ represent the energies of solid, $\left[\mathrm{Co}_{6} \mathrm{Se}_{8}\left(\mathrm{PEt}_{3}\right)_{5}\right]_{2}, \mathrm{C}_{70}$, and toluene molecules, respectively in eqn (1). To investigate further, we have calculated the removal energy of individual components in the solid. The removal energy is computed as the energy difference between the optimized $\left.\left\{\left[\mathrm{Co}_{6} \mathrm{Se}_{8}\left(\mathrm{PEt}_{3}\right)_{5}\right]_{2} \text { (Toluene }\right)_{2} \mathrm{C}_{140}\right\}_{2}$ crystal and the optimized solid without the respective component. We found that the toluene's removal energy is $0.97 \mathrm{eV}$ per molecule, and this comes to $3.88 \mathrm{eV}$ for the four molecules in the unit cell. For comparison, the removal energies of the $\left(\mathrm{C}_{70}\right)_{2}$ and $\left[\mathrm{Co}_{6} \mathrm{Se}_{8}\left(\mathrm{PEt}_{3}\right)_{5}\right]_{2}$ are found to be 9.66 and $7.72 \mathrm{eV}$, respectively. These large removal energies confirm that there is ionic interaction holding the material together. An alternative way of looking at this is that the cohesive energy of the cluster assembled material per electron transfer from the dimer to the $\mathrm{C}_{70}$ is $6.67 \mathrm{eV}$, as there are 4 electron transfers per unit cell. For comparison, the cohesive energy for $\mathrm{NaCl}$ is $\sim 8.19 \mathrm{eV}$ per electron transfer, ${ }^{31,32}$ so the value is in the range expected for an ionic solid. The $6.67 \mathrm{eV}$ cohesive energy combines several factors; in addition to the electron transfer, the bonding that forms the $\left(\mathrm{C}_{70}\right)_{2}$ by combining two $\mathrm{C}_{70}$ is also included, as our reference energy is isolated $\mathrm{C}_{70}$. For free clusters, this bond energy is approximately $1.11 \mathrm{eV}$ for combining two $\mathrm{C}_{70}{ }^{-}$into $\left(\mathrm{C}_{70}\right)_{2}{ }^{2-}$. The result of this dimer formation is a rough estimate since the stability of double anions in free space is much lower than in a solution or in a solid. The cohesive energy of $6.67 \mathrm{eV}$ per electron transfer demonstrates that the present cluster assembled material is not a molecular solid but rather a material held together via ionic bonding.

The projected density of states (PDOS) of metal chalcogenide dimer, fullerene, and toluene within the solid is shown in Fig. 5. The material's bandgap energy is calculated to be $0.24 \mathrm{eV}$, with the valence band edge composed of the states on

Table 1 The charge transfer between fused clusters, $C_{140}$ units, and toluene molecules

\begin{tabular}{lccc}
\hline Units & Charges & Total number of units & Total-Charge \\
\hline Cluster & 0.8056 & 4 & 3.2224 \\
$\mathrm{C}_{140}$ & -1.6174 & 2 & -3.2348 \\
Toluene & 0.00297 & 4 & 0.0119
\end{tabular}




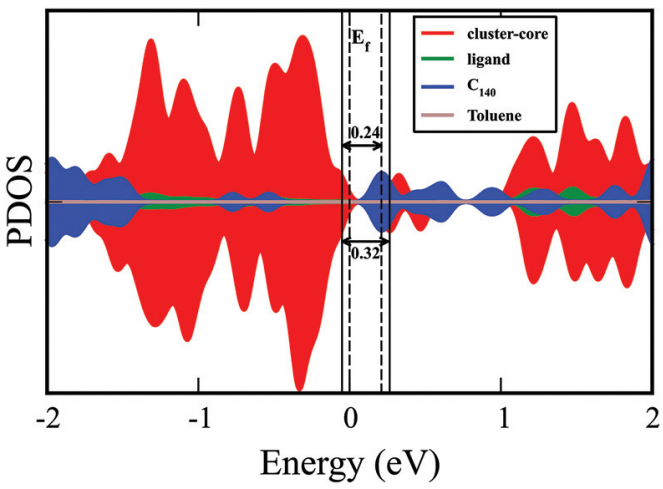

Fig. 5 The projected density of state (PDOS) plot including the various components of $\left.\left\{\left[\mathrm{CO}_{12} \mathrm{Se}_{16}\left(\mathrm{PEt}_{3}\right)_{10}\right]_{2} \text { (Toluene }\right)_{2} \mathrm{C}_{140}\right\}_{2}$ solid.

the metal-chalcogenide dimer. In contrast, the conduction band edge is mostly localized on the fullerenes $\left(\mathrm{C}_{140}\right)$. The gap on the metal-chalcogenide dimers is found to be $0.32 \mathrm{eV}$. The optical bandgap from the experiment is reported to be $0.55 \mathrm{eV}$, which is higher than the calculated value. Our calculated value is expected to be lower than the experimentally observed value since the valence band edge is found on the metal-chalcogenide while the edge of the conduction band is on the fullerenes so that the band edge excitations may be relatively weak. Furthermore, the density functional theory also tends to underestimate the bandgap energy. ${ }^{33-36}$

We next want to understand how the electronic structure changes as we move through the fused cluster dimer and are especially interested in the interfacial region where the two clusters are joined. We plotted the local density of states of different regions of the dimer in Fig. 6. The regions correspond to different cross sections of the fused clusters as we move from one terminal end, the $1^{\text {st }}$ and $2^{\text {nd }}$ regions, through the interface, the $3^{\text {rd }}$ region, and on to the other terminal end of the cluster dimer, the $4^{\text {th }}$ and $5^{\text {th }}$ regions. The $1^{\text {st }}$ and $2^{\text {nd }}$ regions are the mirror images of the $5^{\text {th }}$ and $4^{\text {th }}$ regions,

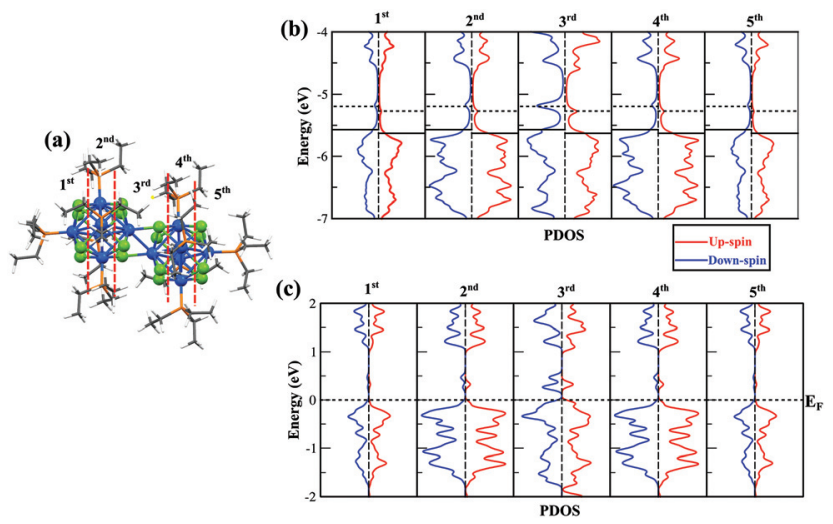

Fig. 6 (a) The splitting of dimer into different regions, (b) local density of states of different sections of the cationic metal-chalcogenide dimer in isolated system and (c) within the $\left\{\left[\mathrm{Co}_{6} \mathrm{Se}_{8}\left(\mathrm{PEt}_{3}\right)_{5}\right]_{2}(\text { Toluene })_{2} \mathrm{C}_{140}\right\}_{2}$ solid. respectively, as shown in Fig. 6a. Fig. 6b provides the local density of states of free cationic dimer $\left[\mathrm{Co}_{6} \mathrm{Se}_{8}\left(\mathrm{PEt}_{3}\right)_{5}\right]_{2}{ }^{+}$, and Fig. $6 \mathrm{c}$ presents the local density of states of the metal-chalcogenide dimer in the cluster assembled solid. In both the free cluster and the solid, we see that the spin is quenched in every region except in the $3^{\text {rd }}$ region that lies at the junction between the fused clusters. In the $3^{\text {rd }}$ region, two unfilled minority states and one unfilled majority impurity states are found, consistent with a spin of $1 \mu_{\mathrm{B}}$ per cluster. This offers additional proof that the spin is localized at the interfacial Co-atoms and that ligands have quenched the spin of Co-atoms at their respective sites. Hence, we have stabilized a ligand protected cluster with a finite magnetic moment at the junction by fusing two clusters. Moreover, we have reconfirmed that the interfacial magnetic moments of the metal-chalcogenide dimers are conserved even after the assembly of the solid.

Next, we consider the effect of adding or removing electrons on the magnetic state of such metal-chalcogenide dimers. The metal-chalcogenide dimers in the solid have +1 charge states with a magnetic moment of $1 \mu_{\mathrm{B}}$. However, the neutral and +2 states of metal-chalcogenide dimers have a magnetic moment of $2 \mu_{\mathrm{B}}$. Hence, if an electron can be added to or taken out from the dimers in the solid, then the magnetic moment of the solid can be increased. Fortunately, the controlled doping of impurities in the crystal lattice is possible through chemical synthesis. ${ }^{30}$ Thus, the addition of electron-donating or electron-withdrawing species to the material might enhance the magnetic moment, offering a cluster assembled material with tunable magnetic properties. ${ }^{37,38}$ It can be used as a method to enhance the magnetic moment in the ligated cluster assembled materials. We would like to add that in recent experiments ${ }^{29}$ the significant electron transport was observed for only one of the three synthesized cluster assembled materials- $\left.\left\{\left[\mathrm{Co}_{6} \mathrm{Se}_{8}\left(\mathrm{PEt}_{3}\right)_{5}\right]_{1.44} \text { (Toluene }\right)_{2} \mathrm{C}_{140}\right\}$, where the distance between the $\mathrm{C}_{140}$ chains was only $3.8 \AA$ (the shortest distance between three cluster assembled materials). It was proposed that the conduction in that assembly was through fullerenes. However, it should be possible to induce conduction pathways through chalcogenide clusters ${ }^{39}$ by appropriate doping. One strategy to induce conductivity through the metal-chalcogen clusters would be to use bidentate aromatic ligands that could connect different clusters or use smaller ligands such as $\mathrm{PMe}_{3}$ to decrease the distance between the clusters. The second strategy could be to use $4 \mathrm{~d}$ or $5 \mathrm{~d}$ transition metals as these clusters may be more delocalized than the $3 \mathrm{~d}$ clusters, resulting in better conduction. However, this may also lead to a change in the spin on the cluster interface. Hence, under circumstances where the conduction is through the metal-chalcogen clusters, the currents will be spinpolarized.

\section{Conclusions}

To summarize, we have shown that by linking two $\mathrm{Co}_{6} \mathrm{Se}_{8}\left(\mathrm{PEt}_{3}\right)_{5}$ clusters, it is possible to form a composite dimer 
$\left[\mathrm{Co}_{6} \mathrm{Se}_{8}\left(\mathrm{PEt}_{3}\right)_{5}\right]_{2}$ that is magnetic. The spin magnetic moment is primarily localized at the interfacial Co-sites, where the two clusters are fused into a dimer. This means that we have stabilized a ligand protected magnetic cluster by combining two highly stable superatomic clusters. Furthermore, the dumbbell shaped dimer is marked by low ionization energy of $4.17 \mathrm{eV}$, which can interact with the similar shaped $\mathrm{C}_{140}$ composed of two $\mathrm{C}_{70}$ units, characterized by a high electron affinity to form a stable ionic solid. Additionally, the theoretical calculations also support the experimental work done by Nuckolls and co-workers, ${ }^{29}$ who have stabilized the anisotropic solids by using toluene molecules as space fillers. Theoretical studies confirm that the $\left[\mathrm{Co}_{6} \mathrm{Se}_{8}\left(\mathrm{PEt}_{3}\right)_{5}\right]_{2}$ dimer loses electrons to $\mathrm{C}_{140}$ in the solid, leaving each metal-chalcogenide dimer as cations. The neutral dimers have a spin moment of $2 \mu_{\mathrm{B}}$ per cluster, while the cationic dimers have spin moments of $1 \mu_{\mathrm{B}}$. When an electron passes through the metal-chalcogenide dimer, the transient neutrality of the cationic species will favor the higher magnetic state of the neutral dimer. Thus, the cluster-dimer is expected to show an intriguing magnetic characteristic when an electron is added to the system. If the electron is induced through the impurities to the cluster assembled materials, it can be used as a strategy to enhance the magnetic moment and might also lead to spin-polarized current. This situation may be useful for spintronic-related applications.

\section{Methods}

\subsection{Theoretical techniques}

The studies performed here are based on the first-principles density functional methods using the Amsterdam Density Functional (ADF) program. ${ }^{40}$ The exchange-correlation functional proposed by Perdew, Burke, and Ernzerhof (PBE), which utilizes the generalized gradient approximation (GGA), was used. ${ }^{41}$ The Slater type valence triple- $\zeta$ basis sets with two polarization functions (TZ2P) basis sets ${ }^{42,43}$ were used for all the elements with a large frozen electron core. The local minimum for each structure was found using the quasi-Newton method ${ }^{44}$ without any symmetry restrictions, and the lowest energy structures were determined for all the calculated systems. The SCF convergence criterion was set to $1 \times 10^{-8}$ Hartree, whereas the default criteria ${ }^{40}$ were used for all optimizations. The relativistic effects were incorporated using the zero-order regular approximation (ZORA). ${ }^{45}$ We have investigated several spin multiplicities to determine the most energetically stable ground state structure for the anionic, neutral, and cationic species.

The geometric optimization and the electronic structure calculation of the periodic crystal structure were performed by the projector-augmented wave (PAW) method ${ }^{46,47}$ as implemented in the Vienna $A b$ initio Simulation Package (VASP). ${ }^{48,49}$ The dispersion-corrected density functional theory (DFT) within the generalized gradient approximation (GGA) framework proposed by Perdew, Burke, and Ernzerhof $(\mathrm{PBE})^{41}$ for the exchange and correlation functional is utilized for all calculations. The DFT-D3 method was used to include the van der Waals corrections. ${ }^{50}$ The Kohn-Sham orbitals were expanded using a plane-wave basis set, and the cutoff was set to $400 \mathrm{eV}$. For the VASP ${ }^{48,49}$ calculations, the crystal structure was fully optimized, and the threshold for the electronic convergence was set to $10^{-5} \mathrm{eV}$, whereas $0.01 \mathrm{eV} \AA^{-1}$ was chosen as the criterion for ionic convergence.

\section{Author contributions}

D.B, A.C.R., and T.S. conducted all the calculations. S.N.K, A.C. R., T.S., and D.B analyzed the results, and the manuscript was written by S.N.K, A.C.R., T.S., and D.B.

\section{Data availability}

The structures and data are given in the ESI. $\dagger$ All additional data generated or analyzed during this study are available via request to the corresponding author.

\section{Conflicts of interest}

The authors declare no competing interests.

\section{Acknowledgements}

The authors gratefully acknowledge funding by the US Department of Energy (DOE) under the award DE-SC0006420.

\section{References}

1 J. F. Corrigan, O. Fuhr and D. Fenske, Adv. Mater., 2009, 21, 1867-1871.

2 C. E. Anson, A. Eichhöfer, I. Issac, D. Fenske, O. Fuhr, P. Sevillano, C. Persau, D. Stalke and J. Zhang, Angew. Chem., Int. Ed., 2008, 47, 1326-1331.

3 C. A. Goddard, J. R. Long and R. H. Holm, Inorg. Chem., 1996, 35, 4347-4354.

4 A. M. Champsaur, J. Yu, X. Roy, D. W. Paley, M. L. Steigerwald, C. Nuckolls and C. M. Bejger, ACS Cent. Sci., 2017, 3, 1050-1055.

5 A. Pinkard, A. M. Champsaur and X. Roy, Acc. Chem. Res., 2018, 51, 919-929.

6 X. Roy, C.-H. Lee, A. C. Crowther, C. L. Schenck, T. Besara, R. A. Lalancette, T. Siegrist, P. W. Stephens, L. E. Brus and P. Kim, Science, 2013, 341, 157-160.

7 B. M. Boardman, J. R. Widawsky, Y. S. Park, C. L. Schenck, L. Venkataraman, M. L. Steigerwald and C. Nuckolls, J. Am. Chem. Soc., 2011, 133, 8455-8457.

8 N. A. Gadjieva, A. M. Champsaur, M. L. Steigerwald, X. Roy and C. Nuckolls, Eur. J. Inorg. Chem., 2020, 2020, 12451254. 
9 C.-H. Lee, L. Liu, C. Bejger, A. Turkiewicz, T. Goko, C. J. Arguello, B. A. Frandsen, S. C. Cheung, T. Medina, T. J. S. Munsie, R. D’Ortenzio, G. M. Luke, T. Besara, R. A. Lalancette, T. Siegrist, P. W. Stephens, A. C. Crowther, L. E. Brus, Y. Matsuo, E. Nakamura, Y. J. Uemura, P. Kim, C. Nuckolls, M. L. Steigerwald and X. Roy, J. Am. Chem. Soc., 2014, 136, 16926-16931.

10 E. J. Telford, J. C. Russell, J. R. Swann, B. Fowler, X. Wang, K. Lee, A. Zangiabadi, K. Watanabe, T. Taniguchi, C. Nuckolls, P. Batail, X. Zhu, J. A. Malen, C. R. Dean and X. Roy, Nano Lett., 2020, 20, 1718-1724.

11 V. Chauhan, A. C. Reber and S. N. Khanna, J. Am. Chem. Soc., 2017, 139, 1871-1877.

12 V. Chauhan, A. C. Reber and S. N. Khanna, J. Phys. Chem. A, 2016, 120, 6644-6649.

13 V. Chauhan, S. Sahoo and S. N. Khanna, J. Am. Chem. Soc., 2016, 138, 1916-1921.

14 A. C. Reber, V. Chauhan and S. N. Khanna, J. Chem. Phys., 2017, 146, 024302.

15 G. Liu, A. Pinkard, S. M. Ciborowski, V. Chauhan, Z. Zhu, A. P. Aydt, S. N. Khanna, X. Roy and K. H. Bowen, Chem. Sci., 2019, 10, 1760-1766.

16 A. C. Reber and S. N. Khanna, Npj Comput. Mater., 2018, 4, 33.

17 A. C. Reber, D. Bista, V. Chauhan and S. N. Khanna, J. Phys. Chem. C, 2019, 123, 8983-8989.

18 G. Liu, V. Chauhan, A. P. Aydt, S. M. Ciborowski, A. Pinkard, Z. Zhu, X. Roy, S. N. Khanna and K. H. Bowen, J. Phys. Chem. C, 2019, 123, 25121-25127.

19 S. T. Akin, V. Zamudio-Bayer, K. Duanmu, G. Leistner, K. Hirsch, C. Bülow, A. Ławicki, A. Terasaki, B. von Issendorff, D. G. Truhlar, J. T. Lau and M. A. Duncan, J. Phys. Chem. Lett., 2016, 7, 4568-4575.

20 S. A. Claridge, A. W. Castleman, S. N. Khanna, C. B. Murray, A. Sen and P. S. Weiss, ACS Nano, 2009, 3, 244-255.

21 S. Mandal, A. C. Reber, M. Qian, P. S. Weiss, S. N. Khanna and A. Sen, Acc. Chem. Res., 2013, 46, 2385-2395.

22 S. Serrano-Guisan, G. di Domenicantonio, M. Abid, J.-P. Abid, M. Hillenkamp, L. Gravier, J.-P. Ansermet and C. Félix, Nat. Mater., 2006, 5, 730-734.

23 A. Turkiewicz, D. W. Paley, T. Besara, G. Elbaz, A. Pinkard, T. Siegrist and X. Roy, J. Am. Chem. Soc., 2014, 136, 1587315876.

24 G. Liu, V. Chauhan, A. P. Aydt, S. M. Ciborowski, A. Pinkard, Z. Zhu, X. Roy, S. N. Khanna and K. H. Bowen, J. Phys. Chem. C, 2019, 123, 25121-25127.

25 A. M. Champsaur, T. J. Hochuli, D. W. Paley, C. Nuckolls and M. L. Steigerwald, Nano Lett., 2018, 18, 4564-4569.

26 A. C. Reber, V. Chauhan, D. Bista and S. N. Khanna, Nanoscale, 2020, 12, 4736-4742.
27 D. Bista, V. Chauhan, T. Sengupta, A. C. Reber and S. N. Khanna, Nanoscale, 2020, 12, 12046-12056.

28 D. Bista, T. Sengupta, A. C. Reber and S. N. Khanna, J. Phys. Chem. A, 2021, 125, 816-824.

29 J. Yang, F. Wang, J. C. Russell, T. J. Hochuli, X. Roy, M. L. Steigerwald, X. Zhu, D. W. Paley and C. Nuckolls, J. Am. Chem. Soc., 2020, 142, 11993-11998.

30 A. Kramida, Y. Ralchenko and J. Reader, NIST Atomic Spectra Database (Ver. 5.7. 1), National Institute of Standards and Technology, Gaithersburg, MD, USA, 2019.

31 M. P. Marder, Condensed matter physics, John Wiley \& Sons, 2010.

32 S. Nagasaka and T. Kojima, J. Phys. Soc. Jpn., 1987, 56, 408414.

33 J. P. Perdew, Int. J. Quantum Chem., 1985, 28, 497-523.

34 J. M. Crowley, J. Tahir-Kheli and W. A. Goddard III, J. Phys. Chem. Lett., 2016, 7, 1198-1203.

35 H. Xiao, J. Tahir-Kheli and W. A. Goddard III, J. Phys. Chem. Lett., 2011, 2, 212-217.

36 H. R. Banjade, J. Pan and Q. Yan, Phys. Rev. Mater., 2021, 5, 014005 .

37 E. S. O’Brien, M. T. Trinh, R. L. Kann, J. Chen, G. A. Elbaz, A. Masurkar, T. L. Atallah, M. V. Paley, N. Patel, D. W. Paley, I. Kymissis, A. C. Crowther, A. J. Millis, D. R. Reichman, X.-Y. Zhu and X. Roy, Nat. Chem., 2017, 9, 1170.

38 S. N. Khanna and A. C. Reber, Nat. Chem., 2017, 9, 1151-1152. 39 J. Yu, C.-H. Lee, D. Bouilly, M. Han, P. Kim, M. L. Steigerwald, X. Roy and C. Nuckolls, Nano Lett., 2016, 16, 3385-3389.

40 G. te Velde, F. M. Bickelhaupt, E. J. Baerends, C. Fonseca Guerra, S. J. A. van Gisbergen, J. G. Snijders and T. Ziegler, J. Comput. Chem., 2001, 22, 931-967.

41 J. P. Perdew, K. Burke and M. Ernzerhof, Phys. Rev. Lett., 1996, 77, 3865-3868.

42 E. Van Lenthe and E. J. Baerends, J. Comput. Chem., 2003, 24, 1142-1156.

43 D. P. Chong, E. Van Lenthe, S. Van Gisbergen and E. J. Baerends, J. Comput. Chem., 2004, 25, 1030-1036.

44 L. Fan and T. Ziegler, J. Chem. Phys., 1991, 95, 7401-7408.

45 E. van Lenthe, J. G. Snijders and E. J. Baerends, J. Chem. Phys., 1996, 105, 6505-6516.

46 P. E. Blöchl, Phys. Rev. B: Condens. Matter Mater. Phys., 1994, 50, 17953.

47 G. Kresse and D. Joubert, Phys. Rev. B: Condens. Matter Mater. Phys., 1999, 59, 1758.

48 G. Kresse and J. Hafner, Phys. Rev. B: Condens. Matter Mater. Phys., 1993, 47, 558-561.

49 G. Kresse and J. Furthmüller, Phys. Rev. B: Condens. Matter Mater. Phys., 1996, 54, 11169-11186.

50 S. Grimme, S. Ehrlich and L. Goerigk, J. Comput. Chem., 2011, 32, 1456-1465. 